\title{
Stabilization of RDT target antigens present in dried Plasmodium falciparum-infected samples for validating malaria rapid diagnostic tests at the point of care
}

Collins Morang' $a^{1,2^{*}}$, Cyrus Ayieko' ${ }^{1}$, George Awinda ${ }^{2}$, Rachel Achilla ${ }^{2}$, Caroline Moseti ${ }^{2}$, Bernhards Ogutu ${ }^{3}$, John Waitumbi ${ }^{2}$ and Elizabeth Wanja ${ }^{4}$

\begin{abstract}
Background: Malaria rapid diagnostic tests (RDTs) are a great achievement in implementation of parasite based diagnosis as recommended by World Health Organization. A major drawback of RDTs is lack of positive controls to validate different batches/lots at the point of care. Dried Plasmodium falciparum-infected samples with the RDT target antigens have been suggested as possible positive control but their utility in resource limited settings is hampered by rapid loss of activity over time.
\end{abstract}

Methods: This study evaluated the effectiveness of chemical additives to improve long term storage stability of RDT target antigens (HRP2, pLDH and aldolase) in dried P. falciparum-infected samples using parasitized whole blood and culture samples. Samples were treated with ten selected chemical additives mainly sucrose, trehalose, LDH stabilizer and their combinations. After baseline activity was established, the samples were air dried in bio-safety cabinet and stored at room temperatures $\left(\sim 25^{\circ} \mathrm{C}\right)$. Testing of the stabilized samples using SD Bioline, BinaxNOW, CareStart, and First Response was done at intervals for 53 weeks.

Results: Stability of HRP2 at ambient temperature was reported at 21-24 weeks while that of PAN antigens (pLDH and aldolase) was 2-18 weeks of storage at all parasite densities. The ten chemical additives increased the percentage stability of HRP2 and PAN antigens. Sucrose alone and its combinations with Alsever's solution or biostab significantly increased stability of HRP2 by $56 \%$ at $2000 \mathrm{p} / \mu \mathrm{L}(\mathrm{p}<0.001$ ). Trehalose and its combinations with biostab, sucrose or glycerol significantly increased stability of HRP2 by $57 \%(p<0.001)$. Unlike sucrose, the stability of the HRP2 was significantly retained by trehalose at lower concentrations (500, and $200 \mathrm{p} / \mu \mathrm{L})$. Trehalose in combination biostab stabilizer increased the percentage stability of PAN antigens by 42 , and $32 \%$ at 2000 and $500 \mathrm{p} / \mu \mathrm{L}$ respectively $(\mathrm{p}<0.01)$. This was also the chemical combination with the shortest reconstitution time $(\sim<20 \mathrm{~min})$.

Conclusions: These findings confirm that stabilizing RDT target antigens in dried P. falciparum-infected samples using chemical additives provides field-stable positive controls for malaria RDTs.

Keywords: Malaria, Rapid diagnostic tests, Chemical additives, Stability, Positive controls

\section{Background}

Malaria is still a leading cause of morbidity and mortality, with 212 million cases and 429,000 deaths reported

\footnotetext{
*Correspondence: collinsmisita@gmail.com

1 Maseno University, P.O Box Private Bag, Maseno, Kenya

Full list of author information is available at the end of the article
}

in 2016 [1]. Rapid diagnostic tests (RDTs) are a major milestone in point-of-care malaria diagnosis in an effort to attain universal access to parasite based diagnosis, consistent with WHO strategies and recommendations [2]. RDTs have several advantages such as ease of use, inexpensive, and real-time diagnosis; but field assessment studies have shown that they can report false results [3]. 
During transportation and storage in tropical climates the quality of RDTs deteriorates due to high temperatures and humidity [4]. Albertini et al. showed that during transport and storage of health commodities, temperatures and humidity can exceed $30{ }^{\circ} \mathrm{C}$ and $94 \%$, respectively in Burkina Faso, Senegal, Ethiopia, and the Philippines [5]. The WHO has set out measures to ensure good manufacturing quality, evaluation programmes for lot testing (pre and post purchase), and provides guidelines for selection of RDTs before purchase [6]. But, lack of proper quality control and quality assurance mechanisms for malaria RDT during transportation and storage remains a major problem [7]. Therefore, there is need to develop positive controls for evaluating the quality of RDTs upon transportation and local storage in health facilities.

Positive control wells (PCW) based on recombinant malaria antigens are the most feasible approach to ensuring quality control of RDT in field operational use [8]. Despite the prospective evaluation of the prototype in a malaria endemic area; they still face challenges such as; validation, long-term storage stability, and technical specifications which are still under development [8]. While the world waits for the complete development of PCW, dried Plasmodium falciparum-infected samples were developed as possible cheaper alternatives in monitoring the performance of RDT in routine use [9]. The dried P. falciparum-infected sample tubes contain the RDT target antigens; histidine rich protein 2 (HRP2), lactate dehydrogenase (LDH) and aldolase $[9,10]$. The challenge facing their applicability is protein degradation and rapid loss of reactivity during prolonged storage at ambient conditions $[9,10]$. Furthermore, it is challenging to establish well characterized samples for specific RDTs, as well as quantification of target antigens. Consequently, there is need to stabilize these target proteins to ensure their conformational stability during transport and storage in typical field conditions.

Protein stabilization involves the prevention of the irreversible loss of the unique chemical structure of the protein [11]. Some of the methods used to stabilize proteins for long-term storage include; ligand stabilization, storage as frozen solutions, salted out precipitates, freeze dried solids, and chemical additive stabilization [12]. Chemical additives prevent the loss of enzymatic activity, prevent denaturation, inhibit irreversible aggregation, and protect proteins against chemical instabilities [13]. The commonly used chemical additives are; salts such as sodium and potassium; as well as organic solvents such as polyethylene glycol, polyethyleneimine and glycerol; and sugars such as trehalose, sorbitol and sucrose [14].

Trehalose stabilizes proteins by various mechanisms, such as vitrification, preferential exclusion, water replacement, hydrogen bonding, and non-specific thermodynamic stabilization [15]. Sucrose stabilizes proteins majorly by preferential exclusion; whereby it increases the chemical potential of the protein which adopts a more stable conformation [16]. Glycerol has also been shown in previous studies to stabilize proteins by interacting preferentially with the hydrophobic parts of the protein structures [17]. Alsever's solution and LDH stabilizer are commercially developed for stabilizing proteins during long term storage $[18,19]$. Biostab enzyme stabilizer has been shown previously to stabilize lysozyme over storage time by preferential hydration on the surface of the protein [20].

This study hypothesized that addition of commercially available chemical additives to dried P. falciparuminfected samples may potentially improve the long-term storage stability of HRP2, pLDH and aldolase. Therefore, this study aimed to investigate the effect of ten chemical additives on the stability of HRP2, pLDH and aldolase in during 53 weeks of storage.

\section{Methods \\ Study design and sample collection}

The study was conducted at the Walter Reed Project, Kisumu a malaria holo-endemic area where transmission occurs throughout the year [21]. The study utilized archived whole blood samples collected from the Kombewa Clinical Research Center under a WRAIR/ KEMRI approved protocol \#1720. Cultured samples were obtained from cryopreserved stocks of $P$. falciparum strains (3D7) maintained at the Walter Reed ProjectKisumu and used to initiate a continuous culture according to the WRAIR/KEMRI approved protocol \#1919. All the collected samples were characterized by expert microscopy and diluted to three experimental parasite densities i.e. 2000,500 , and $200 \mathrm{p} / \mu \mathrm{L}$ using malaria negative group O+ blood. The Group O+ blood was obtained through a USAMRD-K approved study (Scientific Steering Committee; SSC\#1919).

\section{Selection of malaria rapid diagnostic tests (RDT)}

The RDTs were selected based on performance in Round 2 of WHO/FIND/CDC malaria RDT performance evaluations, national guidelines on required performance of RDTs in Kenya, and ability to detect two parasite antigens (WHO/FIND/CDC, 2010). Four different types of RDTs were selected based on panel detection score (PDS) at 200 parasite $/ \mu \mathrm{L}$ of $\geq 90$ (Table 1 ).

\section{Selection of chemical additives}

The selection was based on the literature review on the ability of the chemicals to improve stability of proteins. Additives chosen for this project were polyethylene glycol (Sigma-Aldrich, MO, USA), sucrose (Sigma-Aldrich, 
Table 1 Selected malaria rapid diagnostic tests (RDTs)

\begin{tabular}{|c|c|c|c|c|c|}
\hline Product & Number of bands & Parasite specificity & Manufacturer & Product code & Pf PDS (200 parasites/ $\mu \mathrm{L}$ ) \\
\hline CareStart Malaria HRP2 (Pf) & 2 & Pf & Access Bio, Inc. & G0141 & 99 \\
\hline $\begin{array}{l}\text { First Response Malaria Ag } \\
\text { Combo (PLDH/HRP2) }\end{array}$ & 3 & Pf and Pan & $\begin{array}{l}\text { Premier Medical Corporation } \\
\quad \text { Ltd }\end{array}$ & ॥6FRC30 & 100 \\
\hline $\begin{array}{l}\text { SD BIOLINE Malaria Ag Pf/Pan } \\
\quad(H R P 2 / p L D H)\end{array}$ & 3 & Pf and Pan & Standard Diagnostics, Inc. & 05FK60-02-3 & 96 \\
\hline $\begin{array}{l}\text { BinaxNOW Malaria Ag pf/Pan } \\
\text { (HRP2/Aldolase }\end{array}$ & 3 & Pf and Pan & $\begin{array}{l}\text { Binax, Inc., Inverness Medical, } \\
\text { ME, USA }\end{array}$ & $665-025$ & 99 \\
\hline
\end{tabular}

Pf, Plasmodium falciparum; PDS, parasite detection score; Pan, $\mathrm{pLDH}$ or aldolase antigens

MO, USA), biostab enzyme stabilizer (Sigma-Aldrich, MO, USA), Alsever's Solution (Fisher Scientific, USA), trehalose (Fisher-Scientific, USA), lactate dehydrogenase stabilizer solution (The Gwent Group UK), polyethyleneimine (Fisher-Scientific, USA), and glycerol (SigmaAldrich, MO, USA). Stepwise exploratory tests (36 tests) were conducted to determine which additives, combinations and concentrations gave optimal RDT test outcomes. The tests were conducted by adding the additives to start-up culture samples individually (mono) or in combination (mixed) with other additives at concentrations of 200 and $2000 \mathrm{p} / \mu \mathrm{L}$.

The ratio of additives used in combination was 1:1 (additive: additive) as in previous studies [22]. The ratio of additives or combinations to culture was 1:2 (additive: blood), so as to achieve appropriate concentration of the additive in the blood [23]. A control start-up sample (no additive/s added) was also included. In the event that an additive, combination or concentration was found to interfere with RDT test results, that particular additive or combination was withdrawn. Additives that showed no or little effect on the stability of the proteins were also withdrawn based on weekly retesting results of the samples during 4 weeks of storage on SD Bioline and First Response Malaria Kit. Ten additives/combinations were selected from the 36 combinations (Table 2). The concentration formulations were selected according to extensive previous studies on the effect of different additives on keeping the structure of proteins [14, 24, 25].

\section{Stabilization of Plasmodium proteins}

The ten selected additives and their combinations were added to the patient and culture samples (after characterization to respective parasite densities) in a ratio of $1: 2$, and a control patient and culture sample (without any additive) were also prepared. Baseline data was collected by testing of all the samples to confirm the reactivity of the antigens using SD Bioline, CareStart, First Response and BinaxNOW. The test was conducted according to manufacturer instructions. Reactivity or presence of the HRP2, PLDH, and aldolase was demonstrated by color changes at the test lines. A successful test was confirmed by the presence of a control line. Results were captured as either positive or negative through visual examination of the kit. It was recorded positive if both the control band and the tests bands were visible and it was recorded negative if the control line was visible, but the test lines were absent.

Dried $P$. falciparum-infected samples in tubes were prepared by depositing $40 \mu \mathrm{L}$ aliquots at the bottom of

Table 2 Selected chemical additives for stabilization of malaria RDT target antigens

\begin{tabular}{llll}
\hline Product name & Abbreviation & Concentration used & Manufacturer \\
\hline Sucrose & Suc & $0.5 \mathrm{M}$ & Sigma-Aldrich, MO, USA \\
Glycerol/sucrose & Gly/Suc & $0.5 \mathrm{M}$ and $10 \%$ & Sigma-Aldrich, MO, USA \\
Alsever's/sucrose & Als/Suc & $100 \% \mathrm{w} / \mathrm{v}$ and $0.5 \mathrm{M}$ & Fisher Scientific, USA \& Sigma-Aldrich, MO, USA \\
Trehalose & Treh & $0.5 \mathrm{M}$ & Fisher-Scientific, USA \\
Sucrose/trehalose & Suc/Treh & 0.5 and $0.5 \mathrm{M}$ & Fisher-Scientific, USA \& Sigma-Aldrich, MO, USA \\
Glycerol/Trehalose & Gly/Treh & $10 \%$ and $0.5 \mathrm{M}$ & Fisher-Scientific, USA \& Sigma-Aldrich, MO, USA \\
Trehalose/biostab & Treh/Bio & $0.5 \mathrm{M}$ and $5 \% \mathrm{w} / \mathrm{V}$ & Fisher-Scientific, USA \\
Biostab/sucrose & Bio/Suc & $5 \% \mathrm{w} / \mathrm{v}$ and $0.5 \mathrm{M}$ & Sigma-Aldrich, MO, USA \\
LDH stabilizer & LDH Stab & $100 \%$ W/v & The Gwent Group, UK \\
LDH stabilizer/trehalose & LDH Stab/Treh & $100 \%$ w/v and 0.5 M & Fisher-Scientific, USA \& The Gwent Group, UK \\
\hline
\end{tabular}

$\mathrm{LDH}$, Lactose dehydrogenase; M, Molar; \% W/v, percentage weight/volume 
$2 \mathrm{~mL}$ vials, the tubes were left uncapped to air-dry overnight in a bio-safety cabinet, and then sealed tightly by closing the vial cap. The tubes containing dried P. falciparum-infected sample (stabilized and controls) were stored at room temperature. The temperature of the room was monitored daily, and recorded three times in a day on temperature charts. The average temperature during the study period was $25^{\circ} \mathrm{C}$ (range $23-27{ }^{\circ} \mathrm{C}$ ). Temporal stability data was collected by retesting the dried P. falciparum-infected samples consecutively after 1,4 , $8,12,15,18,21,24,33,43$ and 53 weeks of storage. The study selected 53 weeks of evaluation because, in developing countries like Kenya, there are existing trends of laboratory stock outs in peripheral health facilities due to poor infrastructure and procurement processes [26]. Therefore, stability of antigens for up-to 53 weeks will reduce the chances of facilities having stock-outs, reduce associated costs of resupply, and ensure reliability in using the positive controls.

Retesting was completed by rehydrating the dried blood pellets for the control samples and each additive stabilized sample using phosphate buffered saline with Tween-20. The tubes were left for $1 \mathrm{~h}$ to ensure complete rehydration. Each dried P. falciparum-infected sample was tested on SD Bioline, CareStart, First Response and BinaxNOW. This study defined loss of reactivity as the number of weeks after which a sample tested negative (complete loss of reactivity; $4+$ to 0 ) on a rapid diagnostic test. Potential degradation of RDTs was done on the batch, if a sample was recorded negative (lost reactivity) for any RDT, the reactivity of that batch of RDT was verified using a positive control sample (frozen at $-80^{\circ} \mathrm{C}$ ).

\section{Data analysis}

Stability was defined as the number of weeks the controls or the stabilized sample remained positive as shown by their reactivity on RDTs. The limit of stabilization of a chemical additive is the duration in which the stabilized sample (both culture and patient sample) tested positive for all the three RDTs. CareStart RDT was not included in the analysis because sample retesting could not be conducted to week 53 of storage; due to lack of similar lot number of the kit in the market. Percentage stability of a sample was calculated against week 53 , the endpoint [No. of weeks sample was reactive/53 weeks $\times 100 \%]$. Average percentage stability was computed by combining both the culture and patient samples percentage stabilities. The Z-test for comparing proportions [27] was used to compare the percentage stability of the stabilized sample proteins against the non-stabilized sample proteins. To determine the percentage increase in stability of chemically stabilized HRP2, PLDH, and aldolase, the time taken for a stabilized antigen (in weeks) to lose reactivity on the RDTs was compared to the time taken for non-stabilized antigen to lose reactivity on the RDTs]. The mean percentage increase in stability for each chemical additive was computed from three RDT results for HRP2 or PAN antigens at each parasite density.

\section{Results}

Stability of HRP2, pLDH, and aldolase in non-stabilized dried $P$. falciparum-infected samples

To determine the temporal stability of the RDT target Plasmodium proteins, we measured reactivity of the non-stabilized samples for 53 weeks of storage using three malaria kits. HRP2 from culture and patient samples retained stability for 24 weeks at room temperature as indicated by its reactivity on SD Bioline, First Response, and BinaxNOW malaria RDTs. But, the stability was slightly different (39.6\%) in patient samples when measured with First Response at 2000 and $500 \mathrm{P} / \mu \mathrm{L}$, and BinaxNOW at $500 \mathrm{P} / \mu \mathrm{L}$ (Table 3).

Plasmodium LDH in cultured samples was shown to lose stability in less than 12 weeks. In patient samples, reactivity of pLDH was retained for 12 weeks at $2000 \mathrm{P} /$ $\mu \mathrm{L}$ when evaluated using SD Bioline and First Response. But, stability was shown to reduce as parasite concentration reduced in both patient and culture samples as shown by SD Bioline at $2000 \mathrm{P} / \mu \mathrm{L}(22.64 \%)$ to $11.32 \%$ at $500 \mathrm{P} / \mu \mathrm{L}$ (Table 3).

Plasmodium aldolase was shown to retain stability for 18 weeks of storage at $2000 \mathrm{P} / \mu \mathrm{L}$ as measured by BinaxNOW but reduced to 2 weeks at $500 \mathrm{P} / \mu \mathrm{L}$ in culture samples. The patient samples showed reactivity for aldolase for only 2 weeks of storage because reactivity was not measured for several time points due to lack of similar lot number kits. At $200 \mathrm{P} / \mu \mathrm{L}$, the study reports that PAN antigens were not reactive from baseline analysis in both the culture and patient samples (Table 3).

\section{Stability of HRP2, pLDH, and aldolase present in stabilized dried $P$. falciparum-infected samples}

To determine the effect of chemical additives on the temporal stability of RDT target antigens, we measured reactivity of the stabilized samples for 53 weeks of storage using four RDTs. The limit of stabilization for each chemical additive was shown vary across parasite density levels. Biostab enzyme stabilizer combined with sucrose or trehalose had the best limit of stabilization for 53 weeks across all the parasite densities for HRP2 although their limit of stabilization was lower for PAN antigens (2-24 weeks). Sucrose combined with trehalose had the best limit of stabilization (53 weeks) for PAN antigens at $2000 \mathrm{p} / \mu \mathrm{L}$ but the limit of stabilization was lower (18 weeks) at $500 \mathrm{p} / \mu \mathrm{L}$ (Table 4). 
Table 3 Baseline stability of Plasmodium HRP2, pLDH, and aldolase in non-stabilized samples (study positive controls) during the 53 weeks of analysis

\begin{tabular}{|c|c|c|c|c|c|c|}
\hline \multirow[t]{2}{*}{ Sample type } & \multirow{2}{*}{$\begin{array}{l}\text { Parasite concentra- } \\
\text { tion }\end{array}$} & \multirow[t]{2}{*}{ Malaria RDTs } & \multicolumn{2}{|l|}{ HRP 2} & \multicolumn{2}{|c|}{ pLDH and aldolase } \\
\hline & & & $\begin{array}{l}\text { Reactivity (in } \\
\text { weeks) }\end{array}$ & $\begin{array}{l}\text { Percentage stabil- } \\
\text { ity }\end{array}$ & $\begin{array}{l}\text { Reactivity (in } \\
\text { weeks) }\end{array}$ & $\begin{array}{l}\text { Percentage } \\
\text { stability }\end{array}$ \\
\hline \multirow[t]{9}{*}{ Culture Sample } & \multirow[t]{3}{*}{$2000 \mathrm{P} / \mu \mathrm{L}$} & SD Bioline & 24 & 45.28 & 12 & 22.64 \\
\hline & & First Response & 24 & 45.28 & 12 & 22.64 \\
\hline & & BinaxNOW & 24 & 45.28 & 18 & 33.96 \\
\hline & \multirow[t]{3}{*}{$500 \mathrm{P} / \mu \mathrm{L}$} & SD Bioline & 24 & 45.28 & 6 & 11.32 \\
\hline & & First Response & 24 & 45.28 & 6 & 11.32 \\
\hline & & BinaxNOW & 24 & 45.28 & 2 & 3.77 \\
\hline & \multirow[t]{3}{*}{$200 \mathrm{P} / \mu \mathrm{L}$} & SD Bioline & 24 & 45.28 & 0 & 0.00 \\
\hline & & First Response & 24 & 45.28 & 0 & 0.00 \\
\hline & & BinaxNOW & 24 & 45.28 & 0 & 0.00 \\
\hline \multirow[t]{9}{*}{ Patient Sample } & \multirow[t]{3}{*}{$2000 \mathrm{P} / \mu \mathrm{L}$} & SD Bioline & 24 & 45.28 & 12 & 22.64 \\
\hline & & First Response & 21 & 39.62 & 12 & 22.64 \\
\hline & & BinaxNOW & 24 & 45.28 & 2 & 3.77 \\
\hline & \multirow[t]{3}{*}{$500 \mathrm{P} / \mu \mathrm{L}$} & SD Bioline & 24 & 45.28 & 4 & 7.55 \\
\hline & & First Response & 21 & 39.62 & 2 & 3.77 \\
\hline & & BinaxNOW & 21 & 39.62 & 2 & 3.77 \\
\hline & \multirow[t]{3}{*}{$200 \mathrm{P} / \mu \mathrm{L}$} & SD Bioline & 24 & 45.28 & 0 & 0.00 \\
\hline & & First Response & 24 & 45.28 & 0 & 0.00 \\
\hline & & BinaxNOW & 24 & 45.28 & 0 & 0.00 \\
\hline
\end{tabular}

$\mathrm{P} / \mu \mathrm{L}$, Parasites per microliter; RDTs, rapid diagnostic Tests; $\mathrm{PLDH}$, Plasmodium lactate dehydrogenase; HRP2, histidine rich protein II

\section{Sucrose}

Histidine rich protein II stabilized by sucrose showed $100 \%$ stability at 500 and $2000 \mathrm{p} / \mu \mathrm{L}$, but stability reduced to $62 \%$ at $200 \mathrm{p} / \mu \mathrm{L}$ on all the three RDTs. Combining sucrose with Alsever's solution or biostab enzyme stabilizer enhanced stability of HRP2 at $200 \mathrm{p} / \mu \mathrm{L}$ up to $100 \%$ during the storage period. Combining glycerol with sucrose decreased stability to 81,62 and $40 \%$ at 2000 , 500 and $200 \mathrm{p} / \mu \mathrm{L}$ respectively (Additional file 1: Table $\mathrm{S} 1$ ). The percentage increase in stability of HRP2 was evaluated in the presence of sucrose and its combinations when compared to the non-stabilized samples. Sucrose alone and its combinations with Alsever's solution or biostab stabilizer increased the stability of HRP2 by an average of $56 \%$ ( $p<0.0001$ ), while addition of glycerol increased the stability of HRP2 slightly by $30 \%(\mathrm{p}<0.05)$ at $2000 \mathrm{p} / \mu \mathrm{L})$ (Fig. 1).

Sucrose stabilized PAN antigens in culture samples by $100 \%$ at $2000 \mathrm{p} / \mu \mathrm{L}$, but stability was lower at $81 \%$ in patient samples when measured by SD Bioline and BinaxNOW. Unlike HRP2, combination with Alsever's solution, biostab enzyme stabilizer, or glycerol decreased stability of PAN antigens at $2000 \mathrm{p} / \mu \mathrm{L}$ compared to sucrose alone (Additional file 1: Table S2). There was a significant percentage increase (53\%) in stability of PAN antigens in the presence of sucrose alone than in combination with Alsever's solution (22\%, p < 0.001). Glycerol or biostab stabilizer decreased the stabilizing ability of sucrose significantly on pLDH and aldolase at $2000 \mathrm{p} / \mu \mathrm{L}$, but these combinations increased the stability of the PAN antigens significantly at $(500 \mathrm{p} / \mu \mathrm{L})$ by 29 and $22 \%$ respectively $(\mathrm{p}<0.001)$ (Fig. 2 ). These results indicated that sucrose alone or its combinations had variable impacts on the stability of HRP2, pLDH and aldolase.

\section{Trehalose}

Trehalose alone stabilized HRP2 for the whole storage duration (53 weeks) in concentrations of 2000 and 500 p/ $\mu \mathrm{L}$ but only 43 weeks for $200 \mathrm{p} / \mu \mathrm{L}$. In combination with biostab enzyme stabilizer, the HRP2 retained reactivity on all RDTs (100\% stability) at all three parasite concentrations. When trehalose was combined with sucrose, there was $100 \%$ stability at $2000 \mathrm{p} / \mu \mathrm{L}$, but stability reduced to $45-81 \%$ at $500 \mathrm{p} / \mu \mathrm{L}$ and $45-62 \%$ at $200 \mathrm{p} / \mu \mathrm{L}$. A combination with glycerol reduced the stability further to $40-62 \%$ at lower concentrations despite retaining $100 \%$ stability at $2000 \mathrm{p} / \mu \mathrm{L}$ (Additional file 1: Table S3). Compared to the control samples, the average percentage increase in stability of HRP2 was $56 \%$ at $2000 \mathrm{p} / \mu \mathrm{L}$ in the presence of trehalose and its combinations $(\mathrm{p}<0.0001)$. The percentage increase in stability of HRP2 was significant in the presence of trehalose and in combination 


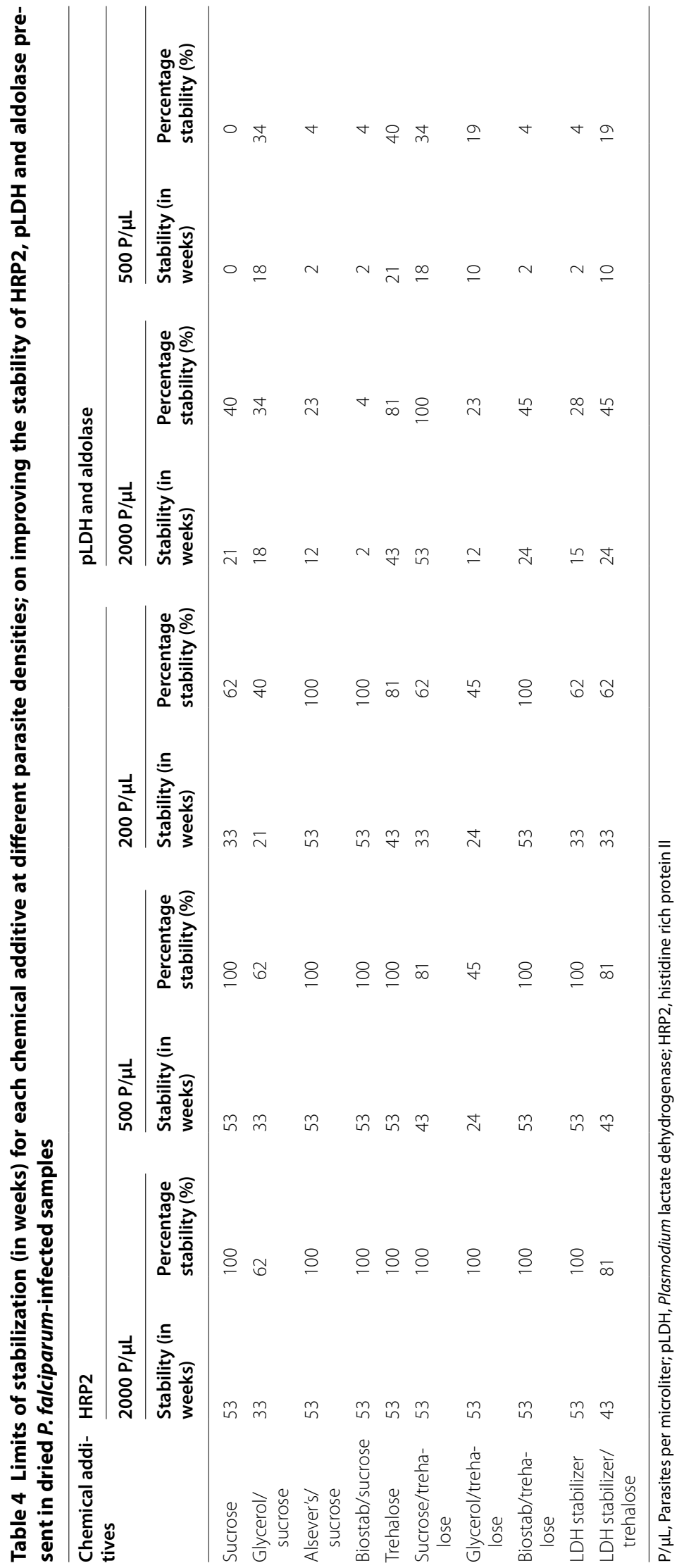




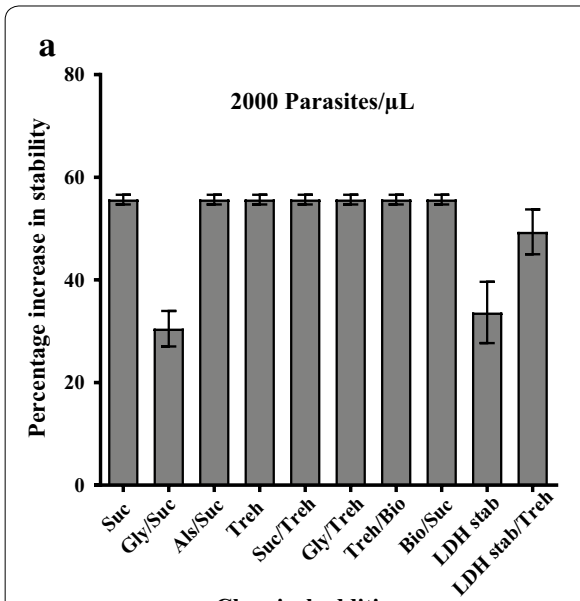

Chemical additives

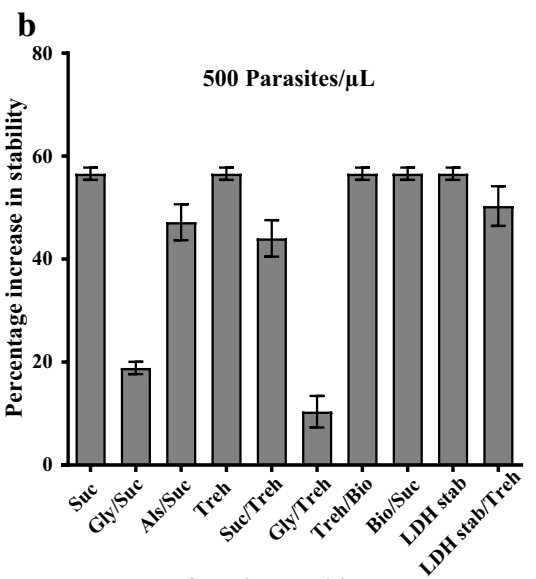

Chemical additives

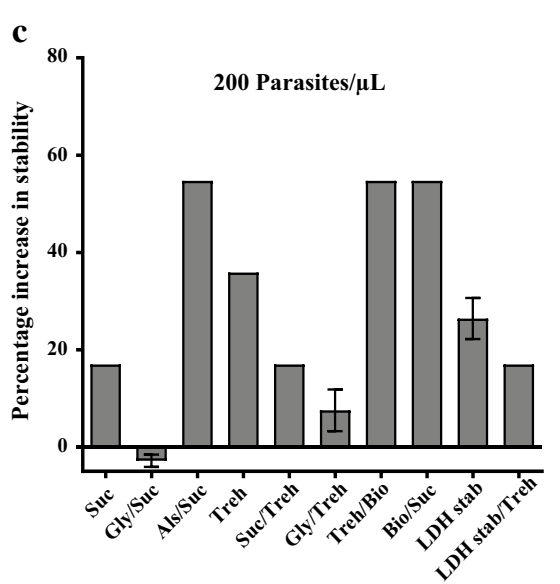

Chemical additives

Fig. 1 Mean percentage increase in stability of HRP2 antigen in stabilized samples (chemical additive added) compared to the control samples (no chemical additive added). Error bars: indicate mean and standard error of the mean. $\mathbf{a}$ The percentage increase in stability of pLDH and aldolase in stabilized samples at $2000 \mathrm{p} / \mu \mathrm{L}$. b Indicates stabilized samples at $500 \mathrm{p} / \mu \mathrm{L}$. c Shows stabilized samples at $200 \mathrm{p} / \mu \mathrm{L}$. Suc sucrose, Gly/Suc glycerol combined with sucrose, Als/Suc Alsever's solution combined with sucrose, Treh trehalose, Suc/Treh sucrose combined with trehalose, Gly/Treh glycerol combined with trehalose, Treh/Bio trehalose combined with biostab enzyme stabilizer, Bio/Suc biostab enzyme stabilizer combined with sucrose, LDH stab lactose dehydrogenase stabilizer, LDH stab/Treh lactose dehydrogenase stabilizer combined with trehalose

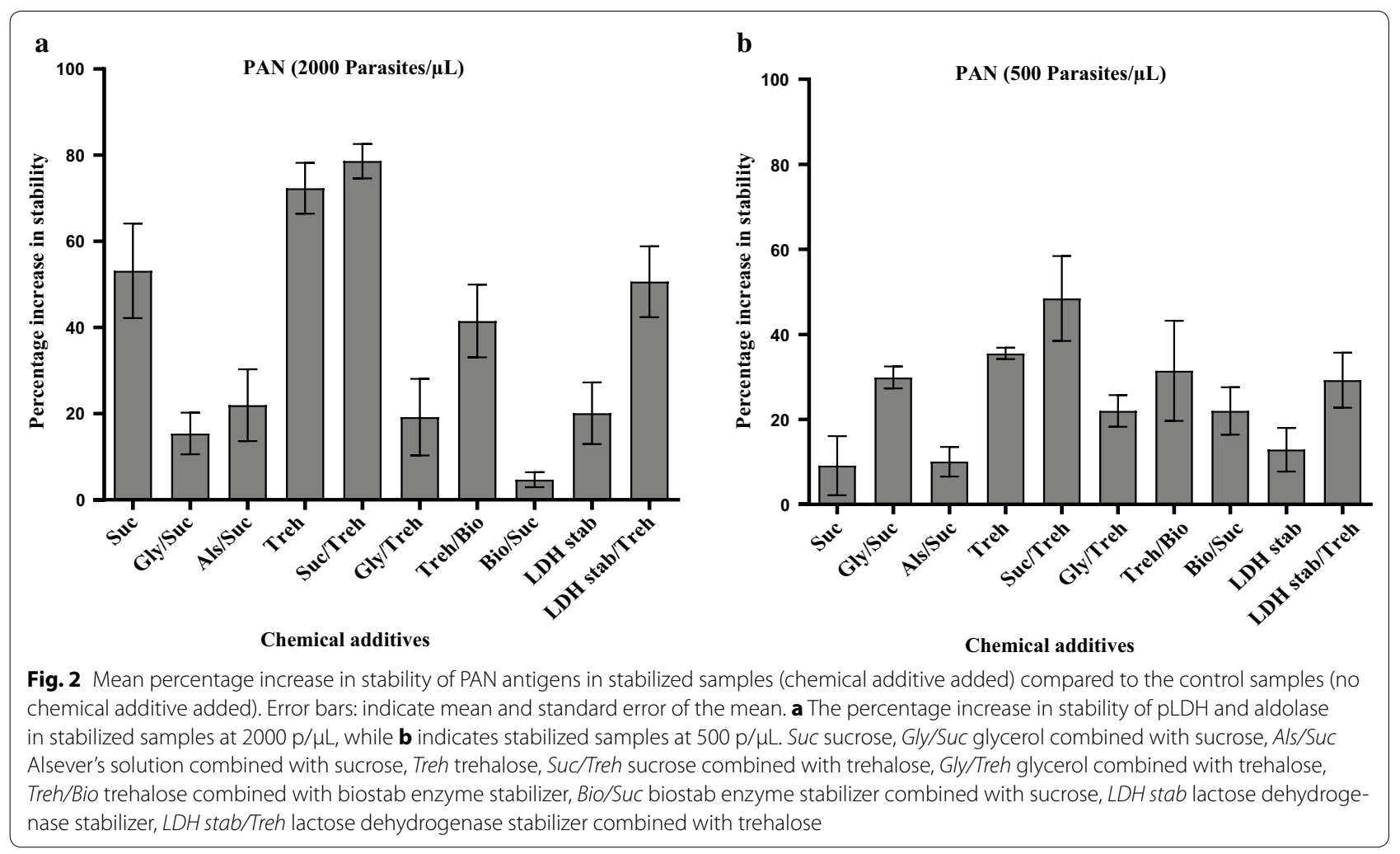

with biostab enzyme stabilizer at both 500 and $200 \mathrm{p} / \mu \mathrm{L}$ ( $\mathrm{p}<0.001)$ (Fig. 1).

In the presence of trehalose alone the PAN antigens retained reactivity for $43-53$ weeks of storage at $2000 \mathrm{p} /$ $\mu \mathrm{L}$, but reactivity was reduced to $40-45 \%$ at $500 \mathrm{p} / \mu \mathrm{L}$.
The combination of trehalose and glycerol had a stability of $23-62 \%$ at $2000 \mathrm{p} / \mu \mathrm{L}$ and $19-34 \%$ at $500 \mathrm{p} / \mu \mathrm{L}$, while the combination with biostab enzyme stabilizer had slightly higher stability of $45-81 \%$ at $2000 \mathrm{p} / \mu \mathrm{L}$ but was highly variable at $500 \mathrm{p} / \mu \mathrm{L}(4-62 \%)$. (Additional file 1 : 
Table S4). There was a significant percentage increase in stability of PAN antigens by $72 \%$ in trehalose alone, $78 \%$ in combination with sucrose, $42 \%$ in combination with biostab enzyme stabilizer, and $19 \%$ in combination with glycerol at $2000 \mathrm{p} / \mu \mathrm{L}(\mathrm{p}<0.0001)$ (Fig. 2).

Trehalose alone and its combination with biostab enzyme stabilizer improved the stability of both HRP2 and PAN antigens consistently throughout the study duration. Of note is that trehalose combination with biostab enzyme stabilizer provided the best chemical additive to improve stability of the dried $P$. falciparuminfected samples due to the ease of reconstitution as compared to trehalose alone.

\section{LDH stabilizer}

Lactate dehydrogenase stabilizer improved the stability ofHRP2 during the storage although there was variability in detection by the three RDTs with stability ranging from $62 \%$ BinaxNOW to $100 \%$ for SD Bioline at $2000 \mathrm{p} /$ $\mu \mathrm{L}$. But the study shows that at lower concentration $(500 \mathrm{p} / \mu \mathrm{L})$, reactivity was retained for the study duration but slightly decreased to $62-81 \%$ at $200 \mathrm{p} / \mu \mathrm{L}$. In combination with trehalose, stability was $81-100 \%$ at both 2000 and $500 \mathrm{p} / \mu \mathrm{L}$ and $62 \%$ at $200 \mathrm{p} / \mu \mathrm{L}$. (Additional file 1: Table S5). This represents a significant percentage increase in stability when compared to control samples (p < 0.001) (Fig. 1).

LDH stabilizer also maintained the stability of pLDH for $12-33$ weeks at $2000 \mathrm{p} / \mu \mathrm{L}$, but reduced to $10-21$ weeks at $500 \mathrm{p} / \mu \mathrm{L}$. The stabilizing ability of LDH stabilizer on aldolase was shown to be minimized from 24 weeks at $2000 \mathrm{p} / \mu \mathrm{L}$ to 2 weeks at $500 \mathrm{p} / \mu \mathrm{L}$. (Additional file 1: Table S6). Addition of trehalose improved the stability of aldolase to 21 and 24 weeks in culture and patient sample respectively. This corresponds to significant increase in average percentage stability by $50 \%$ at $2000 \mathrm{p} / \mu \mathrm{L}$ and $29 \%$ at $2000 \mathrm{p} / \mu \mathrm{L}$ (p < 0.01) (Fig. 2).

\section{Discussion}

This study indicates that chemical additives significantly improve the long-term storage stability of HRP2, $\mathrm{pLDH}$, and aldolase as determined by reactivity on the three malaria RDTs (SD Bioline, First Response, and BinaxNOW). Stabilization of dried P. falciparuminfected samples by sucrose, trehalose, biostab/trehalose, LDH stabilizer/trehalose, and trehalose/sucrose in turn improved the stability of all the three proteins during the 1 year of storage. Previous studies have shown that through preferential interactions these chemical additives improve the long-term stability of various proteins including; ribonuclease A, lysozyme, chymo-trypsinogen recombinant interleukin-1 receptor, monoclonal antibodies, pyro-phosphatase, bovine serum albumin, ribosomal protein S6, cutinase, LDH and lysozyme [12, $15,20,24,25,28-30]$. The findings of the present study show that stabilization of the target proteins ensures prolonged storage of dried Plasmodium falciparum-infected samples in ambient temperature conditions and can be used as positive controls for validation of malaria RDTs at the point of care.

The results on percentage increase in stability clearly indicate that the parasite density was not a factor in conferring stability to the proteins, but loss of reactivity was probably due low levels of the antigens in the samples. As observed, stabilization of the target proteins at $200 \mathrm{p} /$ $\mu \mathrm{L}$ did not prevent their loss of reactivity quicker $(1+$ to 0 ) than samples at $2000 \mathrm{p} / \mu \mathrm{L}(4+$ to 0$)$. Consistent with this findings, Aidoo et al. documented the loss of reactivity in the samples standardized at $200 \mathrm{p} / \mu \mathrm{L}$ as shown by ten different malaria kits was quicker as compared to samples at $2000 \mathrm{p} / \mu \mathrm{L}$ [9]. The present study shows that Plasmodium LDH and aldolase were detectable at baseline by all the four RDTs, but the reactivity of antigens declined quickly with decrease in parasite density during storage. A study by Versteg and Mens indicated that none of the samples containing a parasite density of $300 \mathrm{p} / \mu \mathrm{L}$ gave a signal throughout their study while the samples containing $3000 \mathrm{p} / \mu \mathrm{L}$ were positive for 4 weeks and samples at $30,000 \mathrm{p} / \mu \mathrm{L}$ yielded a signal that remained visible for some time [31].

These results indicate that stability of HRP2, LDH and aldolase increased as concentration of the chemical additives decreased. The percentage temporal stability of all the proteins at $2000 \mathrm{p} / \mu \mathrm{L}$ was lower than $500 \mathrm{p} / \mu \mathrm{L}$ and it increased further at $200 \mathrm{p} / \mu \mathrm{L}$ on SD Bioline, First Response, and BinaxNOW malaria. This can be due to the dilution effect and surface area to volume ratio [32] whereby there is a specific distribution of the additives around the proteins at a particular concentration and size of the protein. In theory, if the protein concentration is reduced and the same amount of the additive is maintained the proteins achieve more stability because all the binding sites of the protein will be saturated depending on the molecular weight. A previous study on effect of sucrose concentration on protein stability showed that; a 1:1 weight ratio provided a sixfold stabilization toward aggregation for hGH $(22 \mathrm{kD})$, a fourfold stabilization for rHSA (66 kD), and a 20-fold stabilization for IgG1 antibody $(150 \mathrm{kD})$ protein [32].

Glycerol/trehalose and glycerol/sucrose are the only additives shown to destabilize or decrease stability of HRP2, LDH, or aldolase. A previous study has shown that glycerol is also preferentially excluded from the surface of the protein, but the exclusion by glycerol is thermodynamically unsuitable, as it favours the unstructured form of proteins [33]. 
The main challenges observed in this study include; differences in the stabilization of HRP2 antigen versus the PLDH and aldolase antigen, low parasite concentration affecting possible outcomes, and noticeable dissimilarity in test performances between the three malaria kits. The differences between stabilization of the proteins can be due levels of the antigens in the sample. This is because different patients have varying expression profiles of the parasite antigens [34] and LDH/aldolase levels can be very low in patient sample as compared to HRP2. Martin et al. showed that the levels of HRP2 are higher in individuals (approximately sevenfold), and can persist in the bloodstream for more than 2 weeks as compared to the PLDH which cannot be detected in smaller volumes of blood and the clearance time rate is less than 5 days [35].

The noticeable dissimilarity between different RDTs can be due to manufacturer differences, and lot to lot or batch to batch variation. Previous studies on patient samples and WHO panel detection scores have shown that the performance of malaria RDTs varies between different manufacturer brands and between different lots from the same manufacturer $[2,3,6,7,9]$. Manufacturer factors, such as the type of immunoglobulin used for antigen capture, the type of strip used, type of buffer used, the quality of fluorescence particles, and the overall kit development, play a critical role in the variability of results among kits [7]. In field use (health facilities), the RDTs have several limitations, such as decreased and variable sensitivity at lower parasite densities, false negative results, and false positive results $[3,36]$.

\section{Conclusion}

This study demonstrated that the presence of chemical additives in dried $P$. falciparum-infected samples significantly improves the long-term stability ( 53 weeks) of HRP2, PLDH, and aldolase. Specifically, sucrose, trehalose, sucrose/trehalose, biostab/trehalose demonstrated the ability to significantly improve the stability of the RDT target antigens. This study also recommends that future exploration in the field be carried out on the use of biostab/trehalose stabilized dried P. falciparum-infected samples. Stabilized dried P. falciparum-infected samples as RDT positive controls should be sent to off-site facilities in different climatic zones both in Kenya and other countries for testing under ambient temperature conditions in-order to determine their field operational viability.

\section{Additional file}

Additional file 1. Percentage stability of Plasmodium HRP2, pLDH, and aldolase after 53 weeks of storage tested on three malaria RDTs after stabilization using chemical additives.

\section{Authors' contributions}

CM contributed in concept development, study design, execution of the project, data analysis, data interpretation and drafting the manuscript. CA was the University supervisor responsible for intellectual content development, study design, interpretation of data and manuscript drafting. GA, RA, and CM participated in the coordination of the study, performing the assays, data collection, and drafting the manuscript. BO, JW and EW substantially contributed to the conception, study design, supervision, data interpretation and drafting of the manuscript. All authors participated in drafting, review manuscript for publication. All authors read and approved the final manuscript.

\section{Author details}

${ }^{1}$ Maseno University, P.O Box Private Bag, Maseno, Kenya. ${ }^{2}$ United States Army Medical Research Directorate, P.O Box 54, Kisumu 40100, Kenya. ${ }^{3}$ Kenya Medical Research Institute, P.O. Box 54840-00200, Nairobi, Kenya. ${ }^{4}$ United States Army Medical Research Directorate-Armed Forces Research Institute of Medical Sciences, Bangkok 10400, Thailand.

\section{Acknowledgements}

I acknowledge Michael Aidoo for his input during the study conception and MAJ. Derek Monthei, Clement Masakhwe, Allan Lemtudo, Kimita Gathii, and Nickline Kuya for their huge support in carrying out this work.

\section{Competing interests}

The authors declare that they have no competing interests.

\section{Availability of data and materials}

The data supporting the conclusions of this paper are included within the paper. Raw data may be obtained from the corresponding author upon request.

\section{Consent for publication}

This study reports no individual data. This work is to be published with permission of the Director, Kenya Medical Research Institute. Material has been reviewed by the Walter Reed Army Institute of Research. There is no objection to its presentation and/or publication. All authors have read and approved the manuscript for publication.

\section{Disclaimer statement}

Material has been reviewed by the Walter Reed Army Institute of Research. There is no objection to its presentation and/or publication. The opinions or assertions contained herein are private views of the author, and are not to be construed as official or as reflecting true views of the U.S Department of the Army, the U.S Department of Defense, or the U.S. Government. The opinions expressed herein are those of the authors and do not reflect the official policy, position, or opinions of Kenya Medical Research Institute.

\section{Ethics approval and consent to participate}

Scientific and ethical approval for the study was obtained from the Ethical Review Committee and Scientific Steering Committee of the Kenya Medical Research Institute, Nairobi (SSC\#2008) and the Walter Reed Army Research Institute of Human Use Research Committee, Silver Spring, Maryland, USA (WRAIR \#1720.002).

\section{Funding}

This work was supported by Military Infectious Diseases Research Program (MIDRP Proposal Number L0324_13_WR_OC).

\section{Issues relating to journal policies \\ None.}

\section{Publication/submission to another journal}

This manuscript has not been published or submitted to another journal for publication.

\section{Publisher's Note}

Springer Nature remains neutral with regard to jurisdictional claims in published maps and institutional affiliations. 
Received: 4 September 2017 Accepted: 23 December 2017

Published online: 08 January 2018

\section{References}

1. WHO. World malaria report 2016. Geneva: World Health Organization; 2016. http://www.who.int/malaria/publications/world-malariareport-2016/report/en/. Accessed 3 Apr 2017.

2. Murray CK, Gasser RA, Magill AJ, Miller RS. Update on rapid diagnostic testing for malaria. Clin Microbiol Rev. 2008;21:97-110.

3. Wanja EW, Kuya N, Moranga C, Hickman M, Johnson JD, Moseti C, et al. Field evaluation of diagnostic performance of malaria rapid diagnostic tests in western Kenya. Malar J. 2016;15:456.

4. Chiodini PL, Bowers K, Jorgensen P, Barnwell JW, Grady KK, Luchavez J, et al. The heat stability of Plasmodium lactate dehydrogenase-based and histidine-rich protein 2-based malaria rapid diagnostic tests. Trans $\mathrm{R}$ Soc Trop Med Hyg. 2007;101:331-7.

5. Albertini A, Lee E, Coulibaly SO, Sleshi M, Faye B, Mationg ML, et al Malaria rapid diagnostic test transport and storage conditions in Burkina Faso, Senegal, Ethiopia and the Philippines. Malar J. 2012;11:406.

6. WHO/FIND/CDC. Malaria rapid diagnostic test performance. Results of WHO product testing of malaria RDTs: Round 5 (2013). Geneva: World Health Organization; 2014. http://www.who.int/malaria/publications/ atoz/9789241507554/en/. Accessed 10 Jan 2015.

7. Mouatcho JC, Goldring JP. Malaria rapid diagnostic tests: challenges and prospects. J Med Microbiol. 2013;62:1491-505.

8. Bell D, Bwanika JB, Cunningham J, Gatton M, Gonzalez IJ, Hopkins H, et al. Prototype positive control wells for malaria rapid diagnostic tests: prospective evaluation of implementation among health workers in Lao People's Democratic Republic and Uganda. Am J Trop Med Hyg. 2017;96:319-29.

9. Aidoo M, Patel JC, Barnwell JW. Dried Plasmodium falciparum-infected samples as positive controls for malaria rapid diagnostic tests. Malar J. 2012;11:239.

10. Tamiru A, Boulanger L, Chang MA, Malone JL, Aidoo M. Field assessment of dried Plasmodium falciparum samples for malaria rapid diagnostic test quality control and proficiency testing in ethiopia. Malar J. 2015;14:11.

11. Carpenter JF, Manning MC, Randolph TW. Long-term storage of proteins. Curr Protoc Protein Sci. 2002; Chapter 4:Unit 4.6.

12. Balcão VM, Vila MM. Structural and functional stabilization of protein entities: State-of-the-art. Adv Drug Deliv Rev. 2015:93:25-41.

13. Ohtake S, Kita Y, Arakawa T. Interactions of formulation excipients with proteins in solution and in the dried state. Adv Drug Deliv Rev. 2011;63:1053-73.

14. Jain NK, Roy I. Effect of trehalose on protein structure. Protein Sci. 2009;18:24-36.

15. Katyal N, Deep S. Revisiting the conundrum of trehalose stabilization. Phys Chem Chem Phys. 2014;16:26746-61.

16. Lee JC, Timasheff SN. The stabilization of proteins by sucrose. J Biol Chem. 1981;256:7193-201.

17. Vagenende $V$, Yap MG, Trout BL. Mechanisms of protein stabilization and prevention of protein aggregation by glycerol. Biochemistry. 2009;48:11084-96.

18. GwentGroup. Stabiliser solution for lactate dehydrogenase; product information sheet. Issue 1. Gwent Group Website. 2010. http://www. gwent.org/aet_data_sheets/stabilisers/q2090625d18.pdf. Accessed 10 Nov 2014.

19. Lowe ML, Gin JB, Demetriou JA. Stability of erythrocytic enzymes for screening tests. Clin Chem. 1973;19:529-30.

20. Subbaraman LN, Glasier MA, Senchyna M, Jones L. Stabilization of lysozyme mass extracted from lotrafilcon silicone hydrogel contact lenses. Optom Vis Sci. 2005;82:209-14.

21. Sifuna P, Oyugi M, Ogutu B, Andagalu B, Otieno A, Owira V, et al. Health and demographic surveillance system profile: the Kombewa health and demographic surveillance system (Kombewa HDSS). Int J Epidemiol. 2014:43:1097-104.

22. Kreilgaard L, Frokjaer S, Flink JM, Randolph TW, Carpenter JF. Effects of additives on the stability of recombinant human factor XIII during freeze-drying and storage in the dried solid. Arch Biochem Biophys. 1998;360:121-34

23. Hamada H, Arakawa T, Shiraki K. Effect of additives on protein aggregation. Curr Pharm Biotechnol. 2009;10:400-7.

24. Kendrick BS, Chang BS, Arakawa T, Peterson B, Randolph TW, Manning $M C$, et al. Preferential exclusion of sucrose from recombinant interleukin-1 receptor antagonist: role in restricted conformational mobility and compaction of native state. Proc Natl Acad Sci USA. 1997;94:11917-22.

25. Yazdani Y, Mohammadi S, Yousefi M, Shokri F. Preliminary assessment of various additives on the specific reactivity of anti-rhbsag monoclonal antibodies. Avicenna J Med Biotechnol. 2015;7:145-50.

26. Wanja E, Achilla R, Obare P, Adeny R, Moseti C, Otieno V, et al. Evaluation of a laboratory quality assurance pilot programme for malaria diagnostics in low-transmission areas of Kenya, 2013. Malar J. 2017;16:221.

27. Hansen JP. Can't miss: conquer any number task by making important statistics simple. Part 6. Tests of significance (z-test statistic, rejecting null hypothesis, $\mathrm{p}$-value), t test, $\mathrm{z}$ test for proportions, statistical significance versus meaningful difference. J Healthc Qual. 2004;26:43-53.

28. Baptista RP, Pedersen S, Cabrita GJ, Otzen DE, Cabral JM, Melo EP. Thermodynamics and mechanism of cutinase stabilization by trehalose. Biopolymers. 2008;89:538-47.

29. Hedoux A, Willart JF, Paccou L, Guinet Y, Affouard F, Lerbret A, et al. Thermostabilization mechanism of bovine serum albumin by trehalose. J Phys Chem B. 2009;113:6119-26

30. Kawai K, Suzuki T. Stabilizing effect of four types of disaccharide on the enzymatic activity of freeze-dried lactate dehydrogenase: step by step evaluation from freezing to storage. Pharm Res. 2007;24:1883-90.

31. Versteeg I, Mens PF. Development of a stable positive control to be used for quality assurance of rapid diagnostic tests for malaria. Diagn Microbiol Infect Dis. 2009;64:256-60.

32. Chang L, Shepherd D, Sun J, Ouellette D, Grant KL, Tang XC, et al. Mechanism of protein stabilization by sugars during freeze-drying and storage: native structure preservation, specific interaction, and/or immobilization in a glassy matrix. J Pharm Sci. 2005:94:1427-44.

33. Zancan P, Sola-Penna M. Trehalose and glycerol stabilize and renature yeast inorganic pyrophosphatase inactivated by very high temperatures. Arch Biochem Biophys. 2005;444:52-60.

34. Daily JP, Scanfeld D, Pochet N, Le Roch K, Plouffe D, Kamal M, et al. Distinct physiological states of Plasmodium falciparum in malaria-infected patients. Nature. 2007:450:1091-5.

35. Martin SK, Rajasekariah GH, Awinda G, Waitumbi J, Kifude C. Unified parasite lactate dehydrogenase and histidine-rich protein elisa for quantification of Plasmodium falciparum. Am J Trop Med Hyg. 2009;80:516-22.

36. Mahende C, Ngasala B, Lusingu J, Yong TS, Lushino P, Lemnge M, et al. Performance of rapid diagnostic test, blood-film microscopy and pcr for the diagnosis of malaria infection among febrile children from korogwe district, tanzania. Malar J. 2016:15:391.

\section{Submit your next manuscript to BioMed Central and we will help you at every step:}

- We accept pre-submission inquiries

- Our selector tool helps you to find the most relevant journal

- We provide round the clock customer support

- Convenient online submission

- Thorough peer review

- Inclusion in PubMed and all major indexing services

- Maximum visibility for your research

Submit your manuscript at www.biomedcentral com/submit
C BioMed Central 